\title{
Special issue on PODC 2013
}

\author{
Gadi Taubenfeld ${ }^{1}$
}

Published online: 26 October 2015

(C) Springer-Verlag Berlin Heidelberg 2015

This special issue of Distributed Computing is based on four papers that originally appeared, in preliminary and abbreviated form, in the Proceedings of the 32nd Annual ACM Symposium on Principles of Distributed Computing (PODC 2013), held in Montreal, Canada on July 22-24, 2013. The papers were chosen by the Program Committee from the 35 full-length papers presented at the Symposium, based on their quality and representation of the range of topics addressed in the Symposium.

PODC is an annual forum for presentation of research on all aspects of Distributed Computing, including the theory, design, implementation and applications of distributed algorithms, systems and networks. The four selected papers, presented in this issue, give a certain glimpse to the scope of issues and techniques that are considered interesting to members in the PODC community.

Achieving agreement in a distributed system despite failures is a central problem in Distributed Computing. In the first paper, On the complexity of asynchronous agreement against powerful adversaries, by Allison Lewko and Mark Lewko, the authors introduce new techniques for proving lower bounds on the running time of randomized algorithms for asynchronous agreement against powerful adversaries. In particular, they define a strongly adaptive adversary that is computationally unbounded and has a limited ability to corrupt a dynamic subset of processors by erasing their memories. They prove that any algorithm with essentially perfect correctness and termination against the strongly adaptive adversary must have exponential running time.

BitTorrent is one of the most popular peer-to-peer protocols: peers interested in downloading a single file from a

\footnotetext{
Gadi Taubenfeld

tgadi@idc.ac.il

1 The Interdisciplinary Center, Herzliya, Israel
}

distinguished user, the seeder, form a so-called swarm. The BitTorrent protocol can become unstable when peers depart immediately after downloading all pieces of a file. Thus, it has been proposed to bundling swarms together, allowing peers to exchange pieces across different swarms, and it was claimed that such "universal swarms" can increase BitTorrents stability. In the second paper, Stable and scalable universal swarms, by Ji Zhu, the author formally characterizes the stability region of universal swarms and shows that they indeed exhibit excellent properties. In particular, bundling allows a single seeder with limited upload capacity to serve an arbitrary number of disjoint swarms if the arrival rate of peers in each swarm is lower than the seeder upload capacity. The result also shows that the stability region is insensitive to peers upload capacity, piece selection policies and number of swarms.

The classical information dissemination problem in radio networks is the problem of broadcasting a single message to all nodes of the network. In the third paper, Randomized broadcast in radio networks with collision detection, by Mohsen Ghaffari, Bernhard Haeupler and Majid Khabbazian, the authors present a randomized distributed algorithm that in radio networks with collision detection broadcasts a single message in $O\left(D+\log ^{6} n\right)$ rounds, with high probability. This time complexity is interesting because of its optimal additive dependence on the network diameter $D$. The authors also study distributed algorithms for broadcasting $k$ messages from a single source to all nodes.

Consensus and approximate agreement are two fundamental problems in Distributed Computing. In the forth paper, Multidimensional agreement in Byzantine systems, by Hammurabi Mendes, Maurice Herlihy, Nitin Vaidya and Vijay K. Garg, the authors discuss generalizations of these two fundamental problems assuming arbitrary Byzantine failures. Consider a network of $n$ processes, where 
each process inputs a $d$-dimensional vector of reals. All processes can communicate directly with others via reliable FIFO channels. The authors discuss the following two problems: (1) the multidimensional Byzantine consensus problem, for synchronous systems, requires processes to decide on a single $d$-dimensional vector $v \in R^{d}$, inside the convex hull of $d$-dimensional vectors that were input by the non-faulty processes; (2) the multidimensional Byzantine approximate agreement problem, for asynchronous systems, requires processes to decide on multiple $d$-dimensional vectors in $R^{d}$, all within a fixed Euclidean distance $\epsilon$ of each other, and inside the convex hull of $d$-dimensional vectors that were input by the non-faulty processes. The authors present necessary and sufficient conditions for solving each one of these two interesting problems.
In addition to being reviewed, in preliminary form, by the Program Committee of PODC 2013, the papers selected for this special issue were subsequently handled by the journal's editors and refereed according to the standard practices of Distributed Computing. I wish to thank the authors, the referees and the journal's handling editors of these papers. Additional papers invited to the special issue will be published separately.

Gadi Taubenfeld

Program Chair, PODC 2013 